\title{
Determinants of processing for a propositional structure
}

\author{
LEON MANELIS \\ Illinois State University, Normal, Illinois 6176I
}

\begin{abstract}
The main purpose of this study was to characterize differences among propositions within a propositional structure. Propositions were described in terms of degree (the number of other propositions to which each is connected) and in terms of a kind of hierarchical level. Paragraphs having a given structure were tested in three tasks: selection of the most important proposition, free recall, and reading under two orienting instructions. Performance was analyzed according to degree and level of the individual propositions and according to the sequences of propositions within the paragraphs. All of these variables affected performance in various tasks. The results were discussed in terms of the distinction between initial encoding and subsequent recall.
\end{abstract}

Much of the recent work on comprehension has made use of propositional systems for analyzing the meaning of prose (for selective reviews, see Anderson, 1976; Meyer, 1975). Basically, these systems are organized sets of categories for decomposing the meaning of extended prose into smaller units and relating the units to each other. A unit commonly discussed is a "proposition," which corresponds roughly to the meaning of a simple sentence. According to Kintsch's system, for example, the meaning of the sentence, "The Greeks loved beautiful art," would be analyzed into two underlying propositions: (LOVE, GREEK, ART) and (BEAUTIFUL, ART) (e.g., Kintsch, 1974, 1976). For longer sections of prose, there would be many propositions that are related to each other, thus forming complicated structures. A basic concern in the recent literature is to describe such structures and, more specifically, to characterize the relative importance of their components. A common approach has been to describe propositional structures as hierarchies and to identify the relative importance of propositions with hierarchical level. Several investigators have concluded that higher level information is more likely to be included in subjects' recall or in their summaries of prose material than is lower level information (Graesser, 1978; Kintsch, 1976; Meyer, 1975; Rumelhart, 1976; Thorndyke, 1977). However, a difficulty with this general conclusion is that the notion of hierarchy has not been used consistently and is not always defined clearly. For example, in Rumelhart's (1976) and Thorndyke's (1977) work, hierarchical level can be defined in terms of the tree structures generated by phrase-structure grammars for stories. Kintsch, on the other hand, defines hierarchical level in terms of connections between propositions. Propositions are said to be connected if they include some of the same concepts (as in the example above). Once a highest level proposition is selected, the next lower level is defined to include those propositions connected to the highest level, and successively lower levels are defined similarly. In their earlier research Kintsch and his colleagues said that a problem with this approach is that selection of the first proposition is not clearly defined; thus, the assignment of levels would rest on an uncertain. judgment (Kintsch, Kozminsky, Streby, McKoon, \& Keenan, 1975, p 199). However, Kintsch and van Dijk (1978) recently have suggested that there are general rules for making the levels assignment more rigorous.

The main purpose of the research reported here was to consider an alternative way of characterizing the relative importance of units within a propositional structure. The alternative is related to Kintsch's (1974, 1976) approach but avoids the potential problem of the initial judgment. One can consider the structural importance of a proposition in terms of "degree," a concept borrowed from graph theory (e.g., Harary, Norman, \& Cartwright, 1965). The degree of a proposition can be defined as the number of other propositions to which it is connected. The higher the degree of a proposition, the more it would be elaborated by other propositions and thus the more it would seem to be the theme for the whole set. The higher the degree, the more that a proposition would provide connecting links for integrating the others. Further, the others would provide more pathways to it for retrieval. Thus, for several reasons, it seems important to consider the effects of degree.

The strategy of the research reported here was to investigate a simple propositional structure in terms of degree as well as in terms of another characteristic. A diagram of the structure and a sample item are shown in Figure 1. Each proposition consists of a verb and two nouns. The nouns provide the connections between propositions, and, as shown in Figure 1, each proposition is identified by the number located at the node for its verb. The degree of Propositions (P1, P3, P4, P5) is two.

The structure can also be described by using Kintsch's 


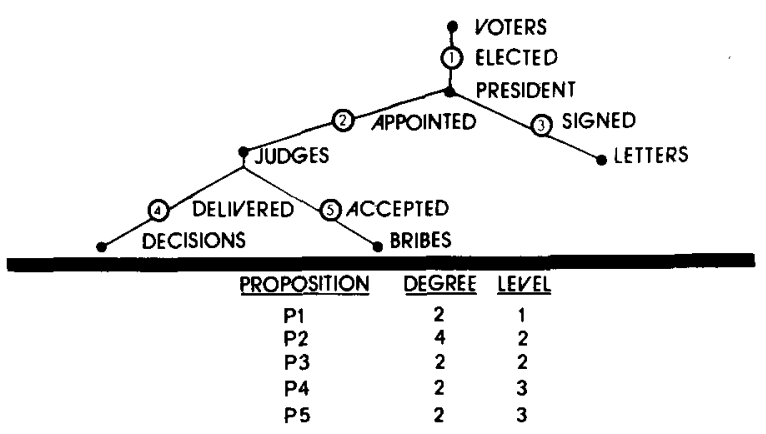

Figure 1. The propositional structure and a sample item.

(1974, 1976) rule for defining hierarchical levels, as presented above. For this purpose, with the present materials there is reason to consider P1 as the first level; according to Kintsch's rule, $\mathrm{P} 2$ and $\mathrm{P} 3$ are then assigned to the second level, and P4 and P5 are assigned to the third. Selection of P1 as the first level (as well as the rest of the assignment) follows the principle that one proposition is at a higher level than another when the object of the one is the same as the subject of the other. As Clark and Haviland (1977) point out, with most simple sentences, the beginning conveys given or presupposed information and the end conveys "new" information, which elaborates on the given. Thus, for the subject-verb-object sentences used here, the levels assignment is reasonable in that the "new "information of a sentence at a particular level is the "given" information for the sentence at the next lower level. ${ }^{1}$ Reflecting this, in the items constructed for the present research, there was a logical and/or chronological sequence across the successive levels, beginning with $\mathrm{Pl}$.

Degree and level provide two different ways of describing propositional structure, as shown in Figure 1. $\mathrm{P} 2$ and $\mathrm{P} 3$, for example, are at the same level but differ in degree; on the other hand, P1, P4, and P5 have the same degree but differ in level. Empirical differences among the propositions might be accounted for in terms of degree of level or both. To investigate empirical differences among the propositions, a set of paragraphs with the structure shown in Figure 1 was tested in three types of tasks. In Experiment 1, the task was to select the proposition most important to the meaning of each paragraph. Experiments 2 and 3 involved recall tests. In Experiment 4 , reading time was measured under two orienting instructions. In all experiments, the sequence of the propositions in the paragraphs varied, and the sequence variable (or the serial position of propositions within sequences) was also examined, as part of the general effort to investigate propositional structure.

\section{EXPERIMENT 1}

The purpose of this experiment was to determine the relative importance of the propositions within the given structure, as subjects judge it deliberately rather than in more naturalistic processing. This task should provide one type of information about how the propositions in the structure are interpreted. In another sense, this experiment was intended to provide some support for using the structure in the first place. Differences among the propositions in judged importance would justify asking how the differences are reflected in the processing tasks involved in Experiments 2 and 3.

\section{Method}

Materials. As described above, each item was a paragraph whose sentences expressed single propositions related according to the structure shown in Figure 1. Each item was written so as to express a coherent narrative about people or animals and episodes in which they could feasibly take part. The sentences were all of the subject-verb-object form. All verbs were in the past tense. Before nouns, only the definite article "the" was used, rather than" $a$ " or "an," in order to avoid problems of reference when the sequence of propositions was varied. In writing the items, seven propositions were included: the five indicated in Figure 1 and two others at Level 3 connected to the object of P3. When an item was presented to subjects, however, there were only five propositions, including two connected to one or the other of the propositions at Level 2, but not both. For the sake of simplicity, however, the higher degree proposition will always be called $\mathrm{P} 2$.

There were 25 items in all. The propositions within the items were ordered according to five different sequences. One sequence was P1-P2-P4-P5-P3; the others followed the same succession of propositions (with P1 after P3) but began with $P 2, P 4, P 5$, and $P 3$, thus producing a Latin square for all five sequences. All 25 items were presented to each subject, and the assignment of sets of 5 items to the five sequences was varied across groups of subjects by following a Latin square. The order of presentation of items was block randomized, with each block containing one item in each sequence.

The items were printed by an IBM line printer. Each item was then placed on one page of a notebook. At the beginning of the notebook were instructions to the subjects, and there was a separate answer sheet for subjects to write all their responses.

Procedure. Subjects were instructed that the sentences on each page of the notebook were related to each other as a short paragraph, but the subjects were not told about the common structure. They were instructed to read each paragraph, select the one that seemed most central to the meaning of the paragraph, and write on the answer sheet the complete sentence they had selected. Subjects were allowed to work through the items at their own pace.

Subjects. Forty psychology students participated in exchange for extra credit in their classes. They were tested in groups of one to five.

\section{Results and Discussion}

The results are shown in Table 1 in terms of the proportion of selections for each proposition. The most common selection was $\mathrm{P} 2$, followed by $\mathrm{P} 1$, and then a tie between $\mathrm{P} 3$ and the mean of P4 and P5. (The latter

Table 1

Proposition of Selections in Experiment 1

\begin{tabular}{lcccc}
\hline & \multicolumn{4}{c}{ Proposition } \\
\cline { 2 - 5 } & P1 & P2 & P3 & P4/5 \\
\hline Proportion of Selections & .25 & .39 & .12 & .12 \\
\hline
\end{tabular}


two have the same position in the structure.) The data were analyzed by the Friedman two-way analysis of variance by ranks, which indicated significant differences among the propositions (with subjects as the unit of replication, $\chi_{\mathrm{r}}^{2}(4)=66.61, \mathrm{p}<.001$; with items as the unit of replication, $\chi_{\mathrm{r}}^{2}(4)=42.46, \mathrm{p}<.001$ ]. [Throughout this paper, both subject and item analyses are reported. Min $\mathrm{F}^{\prime}$ or comparable statistics also are reported when appropriate parametric analyses of variance were performed (Clark, 1973; cf. Wike \& Church, 1976).] In addition to the overall Friedman tests, comparisons among the mean ranks were made by using the procedures outlined by Marascuilo and McSweeney (1967) for the Friedman analysis. An a priori comparison for the hypothesis that the highest degree proposition (P2) was selected more often than the others was significant [for subjects, $\chi^{2}(1)=46.70$, $\mathrm{p}<.001$; for items, $\left.\chi^{2}(1)=32.81, p<.001\right]$. An a priori test of the hypothesis that the Level 1 proposition was selected more often than Level 2, and Level 2 more often than Level 3 was also significant [for subjects, $\chi^{2}(1)=24.06, p<.001 ;$ for items, $\chi^{2}(1)=$ $9.3, \mathrm{p}<.005]$. Post hoc comparisons among all pairs of propositions also indicated differences $(\mathrm{p}<.05)$. In the subject analysis, $\mathrm{P} 1$ and $\mathrm{P} 2$, although not different from each other, were each different from P3, P4, and $\mathrm{P} 5$. In the item analysis, $\mathrm{P} 2$ was different from $\mathrm{P} 3, \mathrm{P} 4$, and P5, but not P1.

The data were also examined according to the serial position of propositions within sequences. (Because the sequences form a Latin square, differences among propositions are orthogonal to differences among serial positions.) There was a slight tendency for the first and last positions to be selected more often than the others, and the fourth position was selected least often. The proportions of selections for the first through fifth positions were, respectively, $.22, .20, .20, .17$, and .21 . Friedman analyses indicated that the effect of position was significant [In the subject analysis, $\chi_{\mathrm{r}}^{2}(4)=10.31$, $\mathrm{p}<.05$; in the item analysis, $\left.\chi_{\mathrm{r}}^{2}(4)=11.77, \mathrm{p}<.02\right]$.

The results of this experiment support the conclusion that degree influences subjects' judgment of the importance of propositions in the structure. However, there is also support for the influence of hierarchical level (as it is defined here). Pitting degree against level might be attempted by comparing P2 (highest degree) with P1 (higher level but lower degree). Although P2 was selected more often, the failure of the post hoc comparisons to differentiate the two makes a general conclusion difficult. Both degree and level were tested further in Experiments 2 and 3.

\section{EXPERIMENT 2}

In this experiment, the items were presented to subjects in a test of free recall. Such memory tests have proved useful in other studies for validating propo- sitional representations (e.g., Kintsch et al., 1975; Meyer, 1975; Thorndyke, 1977). The basic issue is whether hypothesized propositional structures function as memory structures, and one way of testing this--as was done here-is to examine differences in the level of recall among individual propositions. The present experiment also included several study-test trials in order to determine whether the pattern of recall changes at various levels of performance, over the course of learning.

\section{Method}

Materials. The 25 items from Experiment 1 were also used here. In addition, several new items of the same type were included for practice and as fillers. The 25 test items were randomly divided into five sets, and each set was presented to a separate group of subjects. For each group of subjects, the five items in the corresponding set were randomly ordered and were presented to the subjects in that order on five study-test trials. On each trial, a sixth item was added as a filler at the end of the five and before the test in order to eliminate recency. Two items were presented for one study-test trial before the learning trials in order to acquaint subjects with the procedure.

The order of propositions within each paragraph was varied according to the same five sequences as in Experiment 1. Each subject was presented one item in each sequence. Over the whole experiment, each item was presented in every sequence, and each sequence was presented at every one of the five positions (excluding the filler position) within the study trials.

The items for each subject were arranged in a notebook, with one item to a page and a separate blank page for each written recall test.

Procedure. At the outset, subjects were instructed to remember as much as they could and to write their recall in any order. They were also told to write individual sentences or sentence fragments if they could not remember whole paragraphs and to use synonyms if they could not remember exact words.

An audiotape was used for pacing the subjects through the study trials; $15 \mathrm{sec}$ were allowed for each item on each trial, a reading time that had been found sufficient in earlier work. As much time as subjects wanted was allowed for each recall.

Subjects. Fifty psychology students participated in exchange for extra credit in their classes. They were tested in groups of one to five.

\section{Results and Discussion}

The data were analyzed in terms of number of correct propositions. A proposition was scored correct if it was at least a paraphrase of the sentence that had been presented. Changes in the tense of verbs or the number of nouns were ignored.

The proportions of correct propositions are shown in Table 2, classified by proposition type and sequence. The notable feature of the results is that the difference among sequences are larger than the differences among propositions.

Two analyses of variance were computed. In each, proposition, trial, and sequence were factorial independent variables. These analyses provided the opportunity to test for (among other things) a main effect of proposition type as well as any interactions between proposition and sequence or proposition and trial. In the 
Table 2

Proportion of Propositions Correctly Recalled in Experiments 2 and 3

\begin{tabular}{cccccccccc}
\hline & \multicolumn{4}{c}{ Proposition } & \multicolumn{5}{c}{ Sequence } \\
\cline { 2 - 9 } \cline { 6 - 10 } Experiment & P1 & P2 & P3 & P4/5 & $1(12453)$ & $2(24531)$ & $3(45312)$ & $4(53124)$ & $5(31245)$ \\
\hline 2 & .59 & .55 & .57 & .55 & .59 & .54 & .51 & .54 & .64 \\
3 & .35 & .33 & .30 & .34 & .35 & .33 & .28 & .30 & .39 \\
\hline
\end{tabular}

Note-The numbers in parentheses indicate the order of propositions.

first ("subject-item" analysis), the groups of subjects who received the different item sets served as the unit of replication. Following Clark's (1973, 1976) logic, this analysis allowed generalization across both subjects and items. In the second analysis ("subject analysis"), only subjects served as the unit of replication. Both analyses yielded the same conclusions. The only significant effects in the subject-item analysis were sequences and trials [respectively, $\mathrm{F}(4,16)=4.78, \mathrm{p}<.025$, and $F(4,16)=375.69, p<.001]$. Sequences and trials were also the only significant effects in the subject analysis [respectively, $\mathrm{F}(4,180)=4.13, \mathrm{p}<.005$, and $F(4,180)=243.78=243.78, p<.001]$. The effect of propositions did not reach significance in either analysis, and neither did the interaction between proposition and sequence or that between proposition and trial (p > .05). Newman-Keuls follow-up tests for the sequence variable indicated that Sequence 5 produced significantly better performance than Sequences 2, 3 , and $4(p<.05)$. These were the only significant differences, and they were found in both the subjectitem and the subject analyses.

The original purpose of this experiment was to test for the effect of structural position on recall, but the results showed that structural position produced no main effect and no interaction with sequence or trial. The experiment was not totally insensitive, however, because the sequence variable did produce an effect, which will be discussed below. The lack of an effect of structural position might seem at odds with prior research demonstrating recall differences as a function of hierarchical level. However, the present experiment differed from the prior work. Although they all use the loose term "hierarchical level," prior studies have defined hierarchies in four different ways (Graesser, 1978; Kintsch, 1976; Meyer, 1975; Rumelhart, 1976). Thus, it is wrong to conclude that the research by all these investigators implies that an effect of hierarchical level should be obtained in any particular situation without considering the intended definition of hierarchy. In the present study, the definition of hierarchy is closest to that of Kintsch. He and his colleagues have demonstrated effects of hierarchical level several times, but an important difference between that work and the present experiment is the length of the materials. In Kintsch's work on hierarchical level, the materials contained more propositions than the simple five-proposition items used here. There is good reason to believe that simple items need not show a level effect, even though longer items do. Very recently, Kintsch and van Dijk (1978) have articulated a processing theory that could account for differing results depending on length. A central feature of their theory is that hierarchical effects arise from the limited capacity of a short-term memory for propositions. With simple materials that can easily fit the memory capacity, a level effect need not occur. These issues will be considered further in the General Discussion. It should also be noted that memory data do not provide the only test of level or degree as ways of characterizing a propositional structure. Thus, the absence of a memory effect does not invalidate the overall purpose of this study. Experiment 1 provided some initial support for the psychological importance of degree and level, and further support will be provided by Experiment 4 .

Despite the plausibility of finding no structural difference in the memory data of the present experiment, a conclusion of no difference can be somewhat risky. In order to support the conclusion further, a replication experiment will be reported briefly.

\section{EXPERIMENT 3}

The prose materials for this experiment were the same as those used in Experiment 2. The only major difference was that the materials were presented for just one trial, a procedure that has been used more commonly than multitrial learning in memory research on prose. Other procedural differences were that the items were presented on a screen via an overhead projector and that all 25 test items (in addition to the fillers) were presented to each of the 40 subjects rather than only 5 items presented over multiple trials as in the earlier experiment. The results are shown in Table 2. As in Experiment 2, the differences among propositions are minimal and not statistically significant $(p>.05)$. Also as before, the differences among sequences are larger and do show that Sequence 5 produced the best performance $[F(4,156)=9.51, p<.005]$.

\section{EXPERIMENT 4}

Experiments 2 and 3 showed that there are no memory differences among the propositions in the simple structure being tested. However, there was an effect of sequence. The present experiment investigates 
the sequence variable further and will show that differences among the individual propositions do occur when the sequence variable is taken into account.

In Experiments 2 and 3, it was Sequence 5 (shown in Table 2) that produced especially good recall. A characteristic that distinguishes Sequence 5 from the others is that all four pairs of adjacent propositions are connected by repeated words. In the other sequences, only three of the four pairs are connected in this way. The difference of four vs. three does not seem very great, but if the effect of adjacent connections is strong enough, it could have produced the observed results. The present experiment included a test of the effectiveness of adjacent connections. A variety of sequences were grouped into sets that had either a large number of adjacent connections $(\mathrm{C}+)$ or a smaller number $(\mathrm{C}-$ ). Orthogonal to that classification, the sets of sequences differed according to the type of connection that was predominant. In some sets, most of the adjacent connections were the object-to-subject type (OS), in which the object of one sentence is repeated as the subject of the next; in other sets, most of the adjacent connections were subject-to-object (SO). As described above, there is reason to believe that the OS connections will produce better performance. More important, in addition to the classifications by number and type of connections, the design of the experiment allowed the sequences to be compared according to the influence of individual propositions, as will be explained below.

The task in this experiment required subjects to read the items, and the dependent variable was reading time (RT). Half the subjects were instructed to read for the purpose of recalling the items, and the other half were instructed to read for the purpose of producing meaningful elaborations. The instructional variable was included because, in this type of research, an important issue is whether effects generalize from rote artificial to meaningful naturalistic tasks.

\section{Method}

Design and Materials. The 20 sequences that were used here are listed in the Appendix. They were grouped into four sets of five sequences each. Each set formed a Latin square that counterbalanced the individual propositions for serial position. In each of two of the sequence sets $(\mathrm{C}-)$, there was a total of 8 connections between adjacent sentences; in the other two $(\mathrm{C}+)$, there were 16 . All of the eight adjacent connections in one of the $\mathrm{C}$ - sequence sets were $\mathrm{OS}$, and all in the other were SO. In one of the $\mathrm{C}+$ sequence sets, there were eight OS, four subject-to-subject-, and four SO connections. In the other $\mathrm{C}+$ sequence set, there were four $O S$, four subject-to-subject-, and eight SO connections. The four sequence sets thus formed the factorial combinations of number of connections $(C-$ vs. $\mathrm{C}+$ ) and type of connections (predominantly OS vs. SO).

Twenty items from Experiments 1-3 were divided into four sets of five items each. The assignment of item sets to sequence sets was varied across four groups of subjects by following a Latin square.

Each subject was presented one item in each of the 20 sequences and was tested under one of the two orienting instructions. For each collection of 20 items presented, the presentation order was randomized; half the subjects received the items in the randomized order and the other half, in the reverse order. Before the 20 test items, five practice items were presented in order to familiarize subjects with the procedures.

Each item was printed by an IBM line printer and mounted on a card for display in an Iconix four-field tachistoscope with associated timing and control equipment.

Procedure. At the outset of each trial, the subject fixated a marker in a tachistoscope field that was otherwise blank. After the experimenter gave a signal, the subject pressed a start button with the index finger of the left hand whenever he or she was ready. Half a second after the start button was pressed, the fixation field went off and an item appeared, with the first word of the first sentence at the fixation position. All the subjects had been instructed to read each item as quickly as they could while still maintaining good comprehension. They were told to avoid rereading. When finished reading, the subject pressed a stop button with the index finger of the right hand, causing the item to go off and the fixation field to reappear. The time between pressing the start and stop buttons was recorded. After the stop button was pressed, on each trial, the subject did some writing. Half the subjects had been instructed to write as much as they could remember from the item, and the other half had been instructed to write a sentence of their own that was consistent with the meaning of the item but different from any of the sentences they had read.

Subjects. Thirty-two psychology students participated in exchange for extra credit in their classes. They were tested individually.

\section{Results}

Mean RTs are shown in Figure 2 for the factorial combinations of number of connections, type of connection, and orienting instruction. Overall, there was

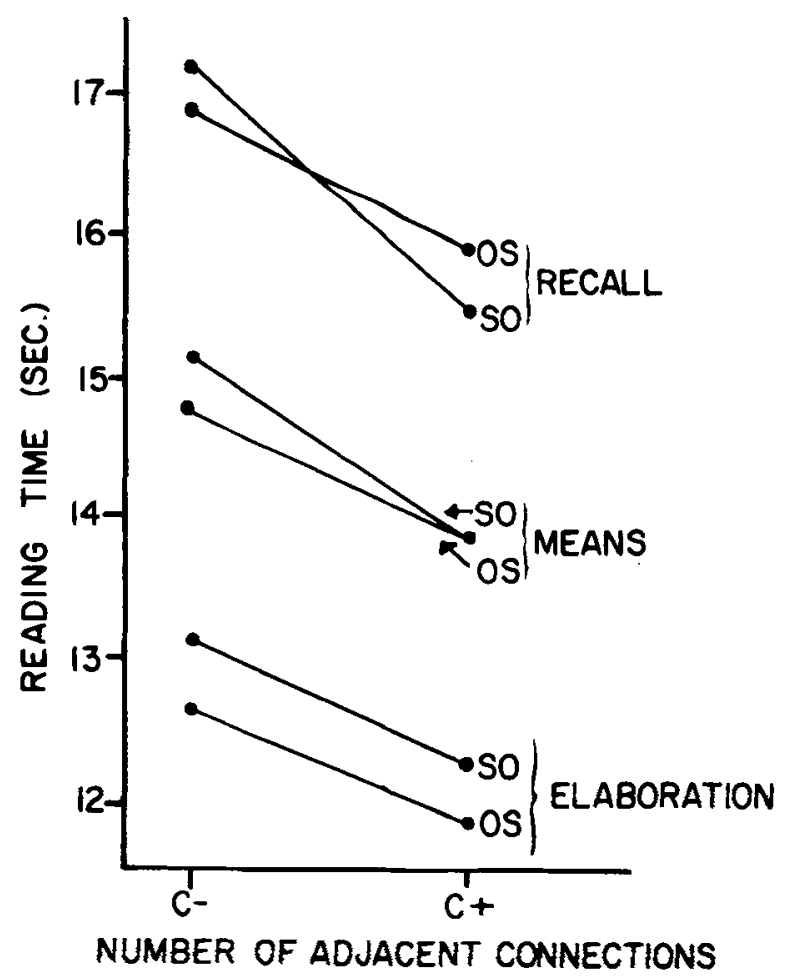

Figure 2. Reading time in Experiment 4, as a function of number of connections, type of connection, and orienting instruction. 
a large difference between the means for $\mathrm{C}-(14.97 \mathrm{sec})$ and $\mathrm{C}+(13.88 \mathrm{sec})$, and there was a large difference between the means for recall $(16.36 \mathrm{sec})$ and elaboration $(12.48 \mathrm{sec})$. Analysis of variance indicated significant effects of number of connections [subject analysis, $F(1,30)=22.71, p<.001$; item analysis, $F(1,19)=6.05$, $\left.\mathrm{p}<.025 ; \min \mathrm{F}^{\prime}(1,29)=4.78, \mathrm{p}<.05\right]$ and instruction [subject analysis, $\mathrm{F}(1,30)=5.18, \mathrm{p}<.05 ;$ item analysis, $F(1,19)=265.53, p<.001 ; \min F^{\prime}(1,31)=5.08$, $p<.05]$. The effect of type of connections and all the interactions among number, type, and instruction were not significant according to the subject analysis, the item analysis, and the combined min $F^{\prime} s(p>.05)$.

The design of the experiment included a Latin square within each sequence set. These Latin squares provided that each proposition occurred equally often at the first serial position within the paragraphs. By appropriately grouping the sequences across sequence sets, comparisons can be made among propositions for that position. That is, for the first serial position, one can compare the RTs when the various propositions in the structure occurred there. (Because equal numbers of RTs for each proposition are drawn from each sequence set, these comparisons are orthogonal to the effects of number and type of connections.) The first serial position has special theoretical importance because it can establish a theme within which the following propositions can be integrated. However, that integration would occur only to the extent that the meaning of the first proposition permits. Propositions that provide better integration thus could be expected to produce faster RTs when they are at the first serial position than when other propositions are at the first serial position.

According to this rationale, then, RT was analyzed as a function of the propositions that occurred at the first serial position. The mean RTs are shown in Figure 3, separately for the subjects given the recall instruction and the subjects given the elaboration instruction. Analysis of variance indicated a significant effect of proposition (in addition to a significant effect of instruction, as reported earlier). Although there was a strong suggestion of an interaction between instruction and proposition, it was not statistically significant. In the subject analysis, the effect of proposition was significant $[F(3,90)=3.37, p<.025]$. The item analysis was complicated by the fact that for the various items, different combinations of propositions but not all of the propositions occurred at the first serial position. The design of the experiment, however, provided that all propositions did occur at the first position, within four sets of five items each. Treating these item sets as the replicate, the effect of proposition was significant $[F(3,9)=4.17, p<.05]$. Computing min $F^{\prime}$ by combining Fs from subject and item analyses was inappropriate because they were based on different statistical designs.

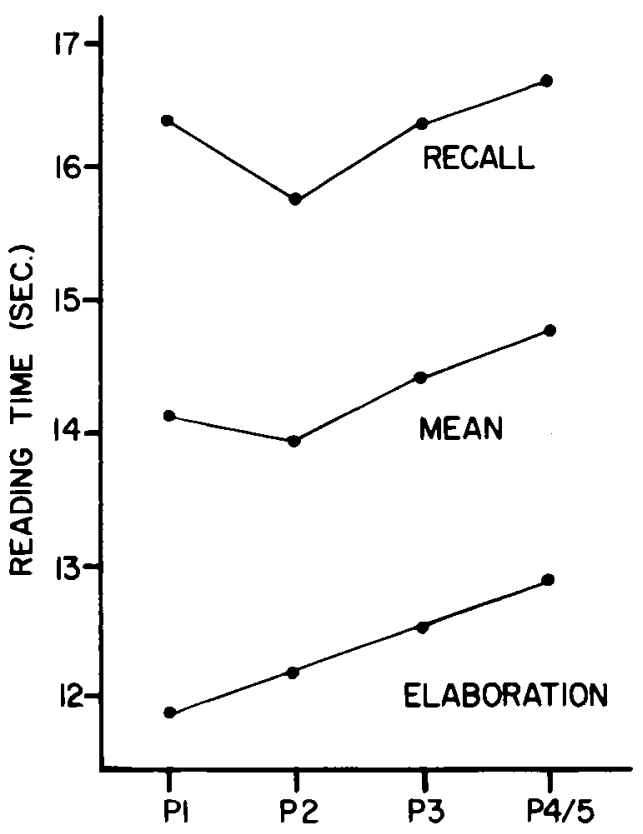

PROPOSITION AT FIRST SERIAL POSITION

Figure 3. Reading time as functions of the proposition at the first serial position in the paragraphs of Experiment 4 .

The pattern of the means for the propositions was very similar to that in Experiment 1 . An a priori test for the hypothesis that the highest degree proposition (P2) produced faster RT than the others was significant [subject analysis, $F(1,90)=4.59, \mathrm{p}<.05$; item analysis, $F(1,9)=6.74, p<.05]$. An a priori test of the hypothesis that the Level 1 proposition had faster RT than Level 2, and Level 2 faster RT than Level 3, was also significant [subject analysis, $F(1,90)=5.51$, $\mathrm{p}<.025$; item analysis, $\mathrm{F}(1,90)=5.76, \mathrm{p}<.05]$. As in Experiment 1, further tests indicated that $P 1$ and P2 were different from the other propositions, according to subject and item analyses $(p<.05)$, but they did not differ from each other.

\section{Discussion}

When classified according to the proposition that occurred at the first serial position, the results of this experiment provide evidence for degree and level as determinants of processing. As shown in Figure 3 and supported by the statistical analyses, all propositions were not equally effective at establishing an integrative theme. The effectiveness of the propositions could be explained according to both level and degree, just as in Experiment 1. The interaction between proposition and instruction was not statistically significant, but in light of the strong suggestion in the pattern of the means, a general conclusion of no interaction would be hasty; if demonstrated reliably in future research, such an interaction between structural position and orienting task would be theoretically important (Aaronson, 1976). ${ }^{2}$ 
A secondary purpose of this experiment was to test the effectiveness of number of connections between the adjacent propositions in a paragraph, a variable that was suggested after observing the results of Experiment 2. The effectiveness of adjacent connections is confirmed by the difference between Conditions Cand $\mathrm{C}+$. That effect did not differ between rote and meaningful orienting instructions. The effect of instructions itself was very large, and at first glance it appears to be in the direction opposite to what might have been expected. Following a similar way of thinking, Craik and Tulving (1975) initially hypothesized that the time to perform an orienting task could be an operational definition of depth of processing (Craik \& Lockhart, 1972) (loosely described as the extent of semantic analysis). Craik and Tulving then rejected that approach (see also Nelson, 1977), and the results of the present experiment provide further evidence for rejecting it because more meaningful processing produced faster RT than did rote processing. The extra time needed for the rote task can be interpreted in two complementary ways. Rote recall demanded storage of many individual words and sentences, whereas meaningful elaboration required storing only a single idea. In addition, meaningful reading is the more automated of the two tasks, and reading for rote recall is likely to require subjects to use strategies they otherwise use relatively infrequently.

\section{GENERAL DISCUSSION}

This study investigated differences among propositions within a propositional structure. The relative importance of the propositions was hypothesized to depend on their degree, the number of other propositions to which any one is connected by virtue of shared concepts. A secondary hypothesis in the present study involved a kind of hierarchical level defined according to Kintsch's $(1974,1976)$ rule of selecting a top level and assigning lower levels based on connections.

Four experiments were conducted. In Experiment 1, clear differences were found among the propositions in the given structure when subjects were asked to judge their relative importance for the meaning of the whole paragraphs. The ordering of the results for the various propositions provided support for both degree and level as determinants of judged importance. In Experiments 2 and 3 , free recall tests were administered in order to determine whether the propositional structure affected memory. No differences among the individual propositions were found, but the sequence in which the propositions were presented did produce an effect. In Experiment 4, the sequence variable was investigated further and showed that the structural position of the propositions is influential: RT varied as a function of the propositions that occurred at the beginning of a paragraph. The ordering of the results for the various propositions was similar to Experiment 1 and again could be explained in terms of both degree and level. Thus, there was support for the role of both structural variables in Experiments 1 and 4. It is reasonable, of course, that both degree and level rather than one or the other can be valid ways of characterizing the processing of propositional structures.

A further question is the nature of the processes that are affected by the characteristics of structure that have been discussed. Experiments 1 and 4 provide support for differences among propositions, whereas Experiments 2 and 3 do not. A basic difference between Experiments 1 and 4 and the others is that the former assessed initial encoding, whereas the others assessed subsequent recall. In Experiments 1 and 4, subjects processed the items while the items were present. In Experiments 2 and 3, on the other hand, subjects were tested after the items had been read. It may be, then, that differences among propositions in degree and level directly influence the initial encoding of a connected structure rather than storage in or retrieval from memory. As suggested by Experiment 4, degree and level seem to determine which propositions are best. for providing an initial theme or anchor to which other propositions can then be connected. This integrative encoding would occur in a short-term memory or work space, but once the encoding is over, although degree and level may affect the encoding time, they need not affect subsequent recall. Degree and level may affect recall for longer passages rather than the short, well-connected paragraphs used here. With longer passages, there would be more material intervening between structurally connected propositions; thus, the difficulty of integrating the propositions within the capacity of a work space would be much greater, and the encoding difficulty could limit memory storage. With short passages, the encoding could be completed, and thus recall effects need not appear. Primarily, then, the present results support the influence of degree and level on initial encoding. Second, the present results provide a kind of specialized test case for a certain interpretation of structural effects on recall when such effects do appear. The present results do not preclude recall effects. Instead, they provide indirect support for the interpretation that, should they occur with longer passages, they are the result of limitations in initial encoding.

This interpretation is entirely consistent with Kintsch and van Dijk's (1978) processing theory. In that theory, recall differences among propositions are explained by the selection of some of them for prolonged maintenance in a short-term buffer. Kintsch and van Dijk speculated that propositions might be selected on the basis of high degree or high level (among other selection criteria). Consistent with their hypothesis, the present results provide evidence that such propositions do play a focal role in immediate processing. 
Finally, the results of this study may be considered in relation to other research on prose. Haviland and Clark (1974) and Manelis and Yekovich (1976) found that sentences or propositions were read faster or remembered better when they were clearly connected by repeated concepts than when they were not. The present study differs from those experiments in that propositions with a fixed pattern of connections were used here, and the number of connections was varied only according to the adjacencies in various sequences. Kieras' (1978) study included tasks similar to the three used here. Primarily, his concern was with the distinction between "good" and "bad" orders of sentences, which he defined as a combination of two criteria. One criterion was coherence, which requires that each sentence be connected with at least one prior sentence. The other criterion was initial topicalization, which requires paragraphs to begin with a main idea, although unlike the present study, Kieras' work was not concerned with defining what a main idea is in general terms. Kieras found differences between good and bad orders, but the two criteria were confounded. The present study has demonstrated effects of connectedness (similar to Kieras' "coherence") in Experiments 2, 3, and 4 with initial sentence type controlled by counterbalancing. It has also demonstrated the effectiveness of initial sentence type (defined specifically by degree and level) in Experiment 4, with connectedness similarly controlled. Thus, Kieras' study, in which the two criteria for goodness of order were confounded, confirms the present study, which manipulated similar characteristics separately and showed that each has its own effects.

A secondary concern in the Kieras (1978) study was to examine differences among individual sentences, the primary concern of the present study. Kieras assumed that one of the sentences in his paragraphs was the theme, a sentence that (in the terms used here) had the highest degree, but it was also different from the others in construction: It was the only sentence based on an active verb rather than on static descriptions, and it contained two nouns, whereas some of the others contained only one. Thus, the designation of the theme was based on construction and degree in a certain combination. Kieras did find some differences among his sentence types; in particular, the theme sentence was selected as an appropriate title for his paragraphs more often than the others. In the present study, however, construction and degree were not confounded because the construction of all sentences was the same. Thus, although Kieras' results are compatible with those found here, the present study introduced the concept of degree and confirmed that it is an effective variable in itself.

In summary, the present study extends the substantial contributions of Kieras, Kintsch, and others who have studied propositional structure by showing that the processing of propositions is related to certain individually defined characteristics of structure: degree and level. Rather than describing structure in terms of combinations of characteristics, however, the aim of the present research was to define individual structural characteristics in terms of some simple but general properties that can easily be subjected to further tests.

\section{REFERENCE NOTE}

1. Walker, C. H., Yekovich, F. R., \& Blackman, H. S. Prerequisites for the formation of linguistic argument repetition. Paper presented at the annual meeting of the Psychonomic Society, St. Louis, Missouri, 1976.

\section{REFERENCES}

Aaronson, D. Performance theories for sentence coding. Journal of Experimental Psychology: Human Perception and Performance, 1976, 2, 42-55.

Anderson, J. R. Language, memory, and thought. Hillsdale, N.J: Erlbaum, 1976.

Clark, H. H. The language-as-fixed-effect fallacy: A critique of language statistics in psychological research. Journal of Verbal Learning and Verbal Behavior, 1973, 12, 335-359.

Clark, H. H. Discussion of Wike and Church's comments. Journal of Verbal Learning and Verbal Behavior, 1976, 15, 257-260.

Clark, H. H., \& Haviland, S. E. Comprehension and the givennew contract. In R. O. Freedle (Ed.), Discourse production and comprehension. Norwood, N.J: Ablex, 1977.

Craik, F. I. M., \& Lockhart, R. S. Levels of processing: A framework for memory research. Journal of Verbal Learning and Verbal Behavior, 1972, 11, 671-684.

Craik, F. I. M., \& Tulving, E. Depth of processing and the retention of words in episodic memory. Journal of Experimental Psychology: General, 1975, 104, 268-294.

Graesser, A. C. How to catch a fish: The memory and representation of common procedures. Discourse Processes, 1978, 1, 72-89.

Harary, F., Norman, R. Z., \& Cartwright, D. Structural models: An introduction to the theory of directed graphs. New York: Wiley, 1965.

Haviland, S. E., \& Clark, H. H. What's new? Acquiring new information as a process in comprehension. Journal of Verbal Learning and Verbal Behavior, 1974, 13, 512-521.

KIERAS, D. Good and bad structure in simple paragraphs: Effects on apparent theme, reading time, and recall. Journal of Verbal Learning and Verbal Behavior, 1978, 17, 13-28.

$\mathrm{KINTSCH}, \mathrm{W}$. The representation of meaning in memory. Hillsdale, N.J: Erlbaum, 1974.

Kintsch, W. On comprehending stories. In E. N. Cofer (Ed.), The structure of human memory. San Francisco: Freeman, 1976.

Kintsch, W., Kozminsky, E., Streby, W. L., McKoon, G., \& KeEnAN, J. M. Comprehension and recall of text as a function of content variables. Journal of Verbal Learning and Verbal Behavior, 1975, 14, 196-214.

KinTSCH, W., \& vaN DiJK, T. A. Toward a model of text comprehension and production. Psychological Review, 1978, 85, 363-394.

Manelis, L., \& Yekovich, F. R. Repetition of propositional arguments in sentences. Journal of Verbal Learning and Verbal Behavior, 1976, 15, 301-312.

Marasciulo, L. A., \& McSweeney, M. Nonparametric post hoc comparisons for trend. Psychological Bulletin, 1967, 67, 401-412. 
MeYer, B. J. F. The organization of prose and its effects on memory. Amsterdam: North-Holland, 1975.

Nelson, T. O. Repetition and depth of processing. Journal of Verbal Learning and Verbal Behavior, 1977, 16, 151-171.

RUMELHART, D. E. Understanding and summarizing brief stories. In D. LaBerge \& S. J. Samuels (Eds.), Basic processes in reading: Perception and comprehension. Hillsdale, N.J: Erlbaum, 1976.

Thorndyke, P. W. Cognitive structures in comprehension and memory of narrative discourse. Cognitive Psychology, 1977, 9, 77-110.

WiKe, E. L., \& Church, J. D. Comments on Clark's “The language-as-fixed-effect fallacy." Journal of Verbal Learning and Verbal Behavior, 1976, 15, 249-256.

\section{NOTES}

1. Walker, Yekovich, and Blackman (Note 1) show that the efficiency of object-to-subject connections does not depend on physical proximity of object and subject.

2. The RT measure in this experiment is best interpreted as the time for initial encoding rather than in terms of comprehension. A comprehension measure would also include what subjects take away from the text after it is read, such as information that is remembered or inferred. Still, as a measure of encoding, RT is meaningful in itself, as demonstrated not only in the present experiment but also in the research of others (e.g., Haviland \& Clark, 1974).

Appendix

Sequence Sets for Experiment 3

\begin{tabular}{lllll}
\hline C+, OS & C+, SO & C-, OS & C-, SO \\
\hline 12453 & 35421 & 25341 & 14352 \\
24531 & 54213 & 53412 & 43521 \\
45312 & 42135 & 34125 & 35214 \\
53124 & 21354 & 41253 & 52143 \\
31245 & 13542 & 12534 & 21435 \\
\hline
\end{tabular}

(Received for publication January 22, 1979; revision accepted October $5,1979$. 